\title{
Abordagem das lesões de tendões flexores na zona II
}

\author{
Approach on flexor tendons injuries at zone II
}

Walter Yoshinori Fukushima', Edison Noboru Fujiki', Gustavo Mantovani Ruggiero', Álvaro Baik Cho', Márcio Aurélio Aita', Yussef Ali Abdouni', Carlo Milani'

\section{Resumo}

A restauração de lesões nos tendões flexores da zona Il é um procedimento difícil devido à sua complexidade anatômica, facilidade de formar aderências e dificuldade de estabelecer protocolos de reabilitação que reduzam o número de rupturas. 0 objetivo deste trabalho foi atualizar o tratamento cirúrgico das lesões nos tendões flexores da zona II. Foram analisados cinco artigos, três sobre avaliação de diferentes técnicas de sutura e dois sobre reabilitação pós-operatória. No estudo de Buendia et al., os valores absolutos mostraram a técnica de ancoragem como sendo a melhor, apesar do desvio padrão ser grande. Já na análise estatística, a ancoragem e o protocolo de Indiana possuem mesma significância. No grupo de imobilização ocorreu tenólise em $21,2 \%$ dos casos sem ruptura tendinosa. Entretanto, no grupo de flexão ativa ocorreu ruptura de tendão em 13,5\% dos casos após duas semanas de mobilização ativa. A sutura de lesão tendinosa na zona II de tendões flexores com quatro passadas apresenta melhores resultados, mas realçamos a importância de analisar a técnica de sutura segundo a resistência, o tempo cirúrgico, a quantidade de material utilizado, 0 aumento do volume, a capacidade traumática e a dificuldade da técnica.

Palavras-chave: Mãos; técnicas de sutura; biomecânica.

\section{Abstract}

The flexor tendon injury repair at zone II is a hard procedure due to its anatomical complexity, easiness in forming adherences and difficulty in establishing rehabilitation protocols to reduce the rupture number. The purpose of this paper was to update the surgical treatment of flexor tendon injuries at zone II. Five articles were analyzed: three about the evaluation of different suture techniques and two about postoperative rehabilitation. In Buendia et al. study, the absolute values demonstrated that the anchorage technique was better than the others, in spite of its greater standard deviation. At statistical analysis, anchorage and Indiana technique had the same significance. In the immobilization group, tenolysis appeared in $21.2 \%$ of the cases without tendon rupture. However, in the active flexion group, tendon ruptures appeared in $13.5 \%$ of the cases, after two weeks of active mobilization. The four strands repair technique for zone II flexor tendon injury presented better results, but we emphasized the suture technique analysis according to its resistance, surgical time, amount of material applied on it, volume increase, trauma capacity and technical difficulty.

Keywords: Hand; suture techniques; biomechanics.

Recebido: 19/5/2009

Revisado: $5 / 10 / 2009$

Aprovado: 16/12/2009

Trabalho realizado na Faculdade de Medicina do ABC (FMABC), Santo André (SP), Brasil

Disciplina de Ortopedia e Traumatologia da Faculdade de Medicina do ABC (FMABC). Santo André (SP), Brasil

Endereço para correspondência: Walter Yoshinori Fukushima - Rua das Aroeiras, 191 - Jardim - CEP 09090-000 - Fone: (11) $4436-3139$ - Santo André (SP) -

E-mail:walterfukushima@ig.com.br 


\section{Introdução}

Lesões causadas nos tendões flexores, principalmente na zona II, resultam em muitos problemas ao paciente por afetarem uma função importante da mão, a preensão. A restauração desse tipo de lesão é bastante complexa em função da dificuldade de acesso, pela presença da bainha sinovial (polias) que envolve os tendões, por meio do processo de aderência que pode se formar durante a cicatrização, e pela necessidade de uma sutura que deve ser ao mesmo tempo resistente e não volumosa.

Com a finalidade de evitar aderências, é preciso preservar uma superfície lisa e promover uma mobilização precoce, o que foi demonstrado cientificamente por Mathews e Richards. Quando cortaram parcialmente o tendão de um coelho dentro do túnel osteofibroso, sem sutura e sem imobilização, observaram que a cicatrização ocorreu sem aderência ${ }^{1}$.

Quanto à reabilitação, Verdan ${ }^{2}$ sugeriu que a mobilização passiva leve por quatro semanas pode romper o reparo. Em 1970, Kleinert et $a l .^{3}$ e Lister $e t ~ a l .{ }^{4}$ relataram os resultados de um reparo do tendão flexor pela sutura de Bunnell, seguida de imediata extensão ativa e flexão passiva com o auxílio de uma banda elástica para prevenir a ruptura pela flexão ativa. Tal protocolo alcançou grande aceitação entre os cirurgiões, sendo reconhecido pelo protocolo de Kleinert ${ }^{3}$.

Entretanto, estudos posteriores mostraram que o tendão deve ser mobilizado por extensão passiva e/ou flexão ativa o quanto antes para prevenir contraturas. Desse modo, o avanço do instrumental, das técnicas comparativas de sutura e do pós-operatório direcionado por protocolos de reabilitação podem tornar a intervenção médica muito bem sucedida. O objetivo é prover atualização no estado da arte do tratamento cirúrgico das lesões em tendões flexores na zona II.

\section{Discussão}

Foram selecionados artigos do período de 1996 a 2009, que versaram sobre os resultados funcionais das técnicas de suturas, protocolos de reabilitação e ensaios básicos experimentais de resistência mecânica de tais técnicas.

Desta maneira, Buendia et al..$^{5}$ utilizaram uma amostra composta por 54 tendões flexores profundos (indicador, médio, anular) de 9 cadáveres frescos, adultos, masculinos, entre 20 e 60 anos, divididos em 3 grupos com 18 tendões cada. Foram aplicadas as técnicas de sutura Kessler modificado, técnicas de ancoragem e método de Indiana. Utilizou-se um fio de propileno 4.0 com sutura combinada de epitendão. Para análise, foi feito um teste de resistência utilizando-se a máquina universal de estudos biomecânicos, com escala de quatro do aparelho (10 kgf; V=20 mm/min), sendo o ensaio filmado. Para análise, foram testados o índice de rigidez, a força máxima da sutura e a formação da separação de $2 \mathrm{~mm}$.

Já o estudo de Severo et al. ${ }^{6}$ foi composto por 84 tendões flexores superficiais e profundos (do polegar ao anular) de seis cadáveres frescos, adultos, entre 15 e 50 anos, divididos em 2 grupos. Em 78 tendões, foram utilizadas 13 técnicas diferentes e os outros 6 tendões restantes ficaram para o grupo controle. Evitou-se o uso de tendões de um mesmo cadáver para uma mesma técnica. O fio utilizado foi de nylon 4.0 na sutura central e, na periferia, 5.0. Para o teste de resistência foi utilizada a máquina de ensaio EMIC-DL $/ 2000(\mathrm{~V}=2 \mathrm{~mm} / \mathrm{min})$.

Em outro estudo, Su et al. ${ }^{7}$ utilizaram 60 tendões flexores digitais profundos da zona II, dissecados das mãos de cadáveres frescos. Apenas os dedos indicador, médio e anular foram utilizados. Após serem seccionados, 30 tendões foram reparados por 3 técnicas distintas: Crucicate com fio 3.0 de poliéster, Cruciate com fio 4.0 e Teno Fix. Os tendões remanescentes foram suturados como os primeiros, e ocorreu a adição de uma sutura circunferencial epitendínea com fio 5.0 de polipropileno monofilamentar. Para o teste de força, foi utilizada a máquina Bionics 858 Material Testing System (MTS Corporation, Eden Praire, MN, USA). A velocidade de separação era de $1 \mathrm{~mm} / \mathrm{s}$ e esta foi monitorada por uma câmera de vídeo digital.

Os artigos de Braga-Silva et al. , $^{8,9}$ sobre mobilização pós-operatória, usufruíram de uma amostra composta por 136 tendões flexores na zona II (secção completa dos tendões flexores superficiais e profundos dos dedos longos e do tendão flexor longo do polegar) de 82 pacientes, adultos, de 19 a 64 anos. Não foram incluídos pacientes com lesão em apenas um dos tendões flexores, lesão multidigital, fraturas, laceração dos extensores, lesão articular e perda de substância (tendinosa, cutânea ou nervosa). Para a tenorrafia, foi aplicada a técnica de Kessler modificada com nylon 3.0, associada a uma sutura epitendinosa contínua com nylon 5.0. A bainha tendinosa foi reparada, mas não suturada. $O$ pós-operatório foi conduzido com a confecção de uma tala gessada dorsal, da extremidade dos dedos até abaixo do cotovelo (punho em, aproximadamente, 30 a $60^{\circ}$ de flexão, metacarpofalangeana em $90^{\circ} \mathrm{e}$ interfalangeana a $0^{\circ}$ ). No estudo randomizado de Braga-Silva et al. ${ }^{9}$, o grupo 1 manteve-se com a tala por três semanas. O grupo 2, assim como em seu outro estudo ${ }^{8}$, adotou o protocolo de mobilização ativa aplicado 12 horas após a cirurgia e envolvia 10 movimentos de extensão passiva e flexão ativa, respectivamente, por hora, durante 16 horas por dia, orientados por profissionais e realizados pelos pacientes sem supervisão. No caso de rerruptura, o paciente era submetido a uma nova tenorrafia com protocolo pós-operatório igual ao da primeira intervenção. A imobilização foi retirada em três semanas e análises foram feitas segundo as classificações da International Federation of Societies for Sugery of the Hand (IFSSH) e de Strickland.

No estudo biomecânico descrito por Buendia et al. ${ }^{5}$, os critérios avaliados foram área do tendão, força máxima aplicada, força de formação da separação de $2 \mathrm{~mm}$ e resistência. Os dados obtidos foram submetidos à análise de variância ANOVA e discriminados pelo teste de Tukey, sendo adotado um nível de significância $(\alpha>5 \%)$. Para interpretação dos resultados, tem-se: grupo 1: Kessler, grupo 2: Ancoragem e grupo 3: Indiana. Em relação à área do tendão, para p=0,1533 e $\mathrm{F}=1,95 \mathrm{~N}$, não foram observadas diferenças estatísticas significativas para as várias técnicas de sutura. 
Foi feita uma análise estatística para a força máxima, com $\mathrm{p}=0,0201$ e $\mathrm{F}=5,62 \mathrm{~N}$ no teste de comparação múltipla. Entre o grupo 1 (Kessler) e o grupo 2 (ancoragem) houve diferença significativa com $\mathrm{p}<0,05$. Entre o grupo 1 (Kessler) e o 3 (Indiana), assim como entre o grupo 2 (ancoragem) e o 3 (Indiana), não houve diferença significativa com $\mathrm{p}>0,05$. Para a força de formação de separação, com $\mathrm{p}=0,0006 \mathrm{e}$ $\mathrm{F}=4,22 \mathrm{~N}$, entre o grupo 1 (Kessler) e o 2 (ancoragem) com $\mathrm{p}<0,001$, e entre o grupo 2 (ancoragem) e o 3 (Indiana) com $\mathrm{p}<0,01$ houve diferenças significativas. Já entre o grupo 1 (Kessler) e o 3 (Indiana) não houve diferença significativa com $\mathrm{p}>0,05$.

O estudo do índice de rigidez demonstrou para Kessler 6,54 $\pm 1,61$

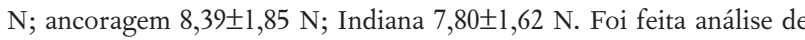
variância com $\mathrm{p}=0,0062$ e F=5,62 N. Houve diferença significativa entre a rigidez 1 (Kessler) e a rigidez 2 (ancoragem) com $\mathrm{p}<0,01$; entre a rigidez 1 (Kessler) e a rigidez 3 (Indiana) e entre a rigidez 2 (ancoragem) e a rigidez 3 (Indiana) não houve significância estatística.

No relato de Severo et al. ${ }^{6}$, foram fixados três parâmetros de resistência medidos em Newtons, os quais são: Fl- início da separação, F2- separação de 3 mm e F3- ruptura completa. Tais parâmetros serviram para testar a variabilidade ou não dos "nós" de cada sutura. Para análise, entre as várias técnicas de sutura, grupo de suturas e deformidades, o teste de Schefee foi escolhido, com nível de 5\% de significância. Em F1 não houve diferença estatística significativa entre as suturas de Pittsburgh, Cruciate, Savage, Yoshizu, Kessler duplo e Brasil-2. O coeficiente de variância foi de 39,64\%. Em F2 não houve diferença estatística significativa entre as suturas de Pittsburgh, Savage, Cruciate, Kessler duplo, Brasil-2, Yoshizu, Brasil-1, Kessler com sutura contínua no epitendão, Indiana, Kleinert modificado de Bunnell e Tsai. O coeficiente de variação foi de $38,28 \%$. Em F3 não houve diferença estatística significativa entre as suturas Pittsburgh, Savage, Cruciate, Kessler duplo, Yoshizu, Brasil-1, Kessler e Tsai. O coeficiente de variação foi de $30,23 \%$.

O estudo de Su et al. ${ }^{7}$ revelou que não houve diferenças significativas entre as espessuras médias de tendões dos dedos indicador, médio e anelar. A média dos tendões foi de $5,9 \mathrm{~mm}$. As técnicas Teno Fix e Cruciate 3.0 apresentaram melhores resultados em relação à técnica Cruciate 4.0, com e sem a sutura circunferencial. Em contrapartida, a técnica Teno Fix obteve melhores resultados sobre as outras duas técnicas, com e sem sutura circunferencial, no quesito energia absorvida.

Em relação à energia absorvida, a técnica Teno Fix mostrou melhores resultados em relação à Cruciate 3.0 e Cruciate 4.0, com ou sem auxílio da sutura circunferencial. Com relação à rigidez, as técnicas Teno Fix e Cruciate 3.0 obtiveram resultados significantemente melhores que Cruciate 4.0. É valido ressaltar que a aplicação de sutura circunferencial melhorou a capacidade de tração de todos os tendões.

Braga-Silva et al. ${ }^{8,9}$ mencionou seus resultados 12 meses após cirurgia, com tempo de acompanhamento de 12 a 36 meses. No primeiro artigo foram analisados, segundo a IFSSH e Strickland, os resultados em dedos longos e polegar, mostrando uma grande aceitação da técnica tanto em dedos longos, quanto no polegar.

Uma análise diferente foi feita no estudo randomizado em BragaSilva et al. ${ }^{9}$. Os resultados foram tabulados e interpretados segundo Mann-Whitney para mobilização ativa e imobilização. A mobilização ativa precoce foi muito superior à imobilização em ambos os sistemas de avaliação ( $p>0,01)$. No grupo 1, a tenólise ocorreu em 9 (21,2\%) casos sem ruptura tendinosa. Entretanto, no grupo 2, ocorram 5 (13,5\%) rupturas de tendões após a segunda semana de mobilização ativa.

Percebe-se dificuldade em associar uma sutura que proponha maior resistência e permita adequado deslizamento, em função da quantidade e variedade de suturas em tendões oferecidas atualmente. Além disso, os protocolos de mobilização interferem diretamente no sucesso ou não da intervenção cirúrgica. Os trabalhos sobre suturas revisados pelos autores deste trabalho são modelos laboratoriais, realizados em cadáveres e testados com máquinas de ensaio, não sendo possível considerar as condições favoráveis à formação de aderências e, desse modo, nos impossibilitando de indicá-las sem restrições. Mesmo assim, eles são válidos, por comprovarem, em valores reais, a força de cada técnica e valorizá-las no meio médico.

Além das técnicas de suturas convencionais aplicadas, o cirurgião atual pode, ainda, dispor da sutura de aço, conhecida por Teno Fix. Tal técnica tem seu valor significativo por ser de aplicação rápida, mantendo o padrão de resistência necessário quando comparado à técnica consagrada do Cruciate. Em contrapartida, ela demanda grande destreza por parte do cirurgião, além de encarecer o procedimento. No entanto, Wolfe et $a l .^{10}$, em seu estudo, não observaram vantagem significativa entre os métodos de Tenofix, Cruciate e Kessler modificado. Em função da divergência de resultados, torna-se difícil acreditar na confiabilidade do estudo de Su et al. .

A segunda fase da intervenção médica é por conta da reabilitação. Sabe-se que longos períodos de imobilização após tenorrafia provocam níveis inaceitáveis de aderência, levando a resultados insatisfatórios. Em função disso, vários protocolos de mobilização precoce foram desenvolvidos, mesmo sabendo-se que podem provocar um índice maior de ruptura no pós-operatório imediato. Young e Harmon ${ }^{11}$, em 1960, baseados na tração dinâmica em flexão, descreveram um programa de mobilização precoce passiva. Nos anos seguintes, os protocolos de Kleinert (flexão passiva e extensão ativa) foram bastante aceitos na reabilitação das lesões de zona II, defendidos por Fernandes et al. ${ }^{12}$ em 1996. A flexão ativa precoce oferece maior risco de ruptura, mas, ao mesmo tempo, pode apresentar vantagens sobre a flexão passiva. $\mathrm{O}$ trabalho de Braga-Silva et al. ${ }^{9}$, em seus resultados, mostra que a imobilização é capaz de levar a tenólise (21,2\%), enquanto na flexão ativa somente 13,5\% rompeu após a segunda semana. A preocupação com a deiscência precoce é a principal razão pela qual os cirurgiões não optam pela flexão ativa. Desse modo, o estudo realizado por Braga-Silva et al. . $^{8,9}$ apresenta resultados satisfatórios, porém difíceis de serem aplicados com segurança, uma vez que a flexão ativa leva a uma porcentagem significativa 
de ruptura. Vale ressaltar que seu trabalho comparou o protocolo de imobilização pelo uso dessa conduta em vários locais, principalmente nos que operam pelo Sistema Único de Saúde (SUS) do Brasil, o qual possui sistema deficiente no acompanhamento pós-operatório. Assim, quando o paciente não é cooperativo com regime de mobilização precoce, seja por falta de acompanhamento profissional e/ou de um esclarecimento ou vontade pessoal, ocorre queda em sua reabilitação.

Os relatos dos estudos privilegiam a quantificação dos resultados, porém é importante ressaltar que cada técnica de sutura deve ser anali- sada pelo tempo de duração da cirurgia, quantidade de material utilizado, aumento de volume do tendão e potencial traumático.

Desta maneira, as técnicas de quatro passadas são as mais indicadas, em razão de os critérios mecânicos de força, rigidez e energia de absorção estarem presentes e propiciarem um melhor resultado cirúrgico.

Em relação ao pós-operatório, fica evidente que a mobilização precoce dos dedos deve ser procedimento adotado com vistas a evitar formação de aderências que prejudicam o deslizamento dos tendões.

\section{Referências}

1. Pardini AG. Traumatismos da mão. 3a ed. Rio de Janeiro: Medsi; 2000.

2. Verdan CE. Primary repair of flexor tendons. J Bone Joint Surg Am. 1960;42-A: 647-57.

3. Kleinert HE, Kutz JE, Atasoy E, Stormo A. Primary repair of flexor tendons. Orthop Clin North Am. 1973:4(4):865-76.

4. Lister GD, Kleinert HE, Kutz JE, Atasoy E. Primary flexor tendon repair followed by immediate controlled mobilization. J Hand Surg Am. 1977;2(6):441-51.

5. Buendia LA, Junior RM, Ulson HJR. Estudo biomecânico comparativo da resistência à tração entre técnicas de suturas dos tendões flexores da mão. Rev. bras. ortop. 2005;40(7):407-17.

6. Severo AL, Ávila AOV, Costa M, Lech OLC. Reparo de tendões flexores da mão: análise biomecânica com diferentes técnicas de sutura. Rev. bras. ortop. 2005;40(7):418-27.

7. Su BW, Protopsaltis TS, Koff MF, Chang KP, Strauch RJ, Crow SA et al. The biomechanical analysis of a tendon fixation device for flexor tendon repair.
J Hand Surg Am. 2005;30(2):237-45

8. Braga-Silva J, Calcagnotto G, Oliveira CG, Fisher H. Estudo prospectivo randomizado da mobilização ativa precoce após reparo de tendões flexores na zona 2. Rev. bras. ortop. 2003;38(10):581-8.

9. Braga-Silva J, Martins PDE, Román J, Gehlen D. Mobilização pós-operatória com flexão ativa precoce após reparo de tendões flexores na zona 2. Rev. Soc. Bras. Cir. Plást. 2005;20(4):207-12.

10. Wolfe SW, Willis AA, Campbell D, Clabeaux J, Wright TM. Biomechanic comparison of the Teno Fix tendon repair device with the cruciate and modified Kessler techniques. J Hand Surg Am. 2007;32(3):356-66.

11. Young RE, Harmon JM. Repair of tendon injuries of the hand. Ann Surg. 1960;151:562-6

12. Fernandes $\mathrm{CH}$, Matsumoto MH, Santos JBG, Araújo PMP, Fallopa F, Albertoni WM. Resultados das tenorrafias em flexores dos dedos da mão, na zona II, submetidos a movimentação precoce passiva assistida. Rev. bras. ortop. 1996:31(6):497-501. 